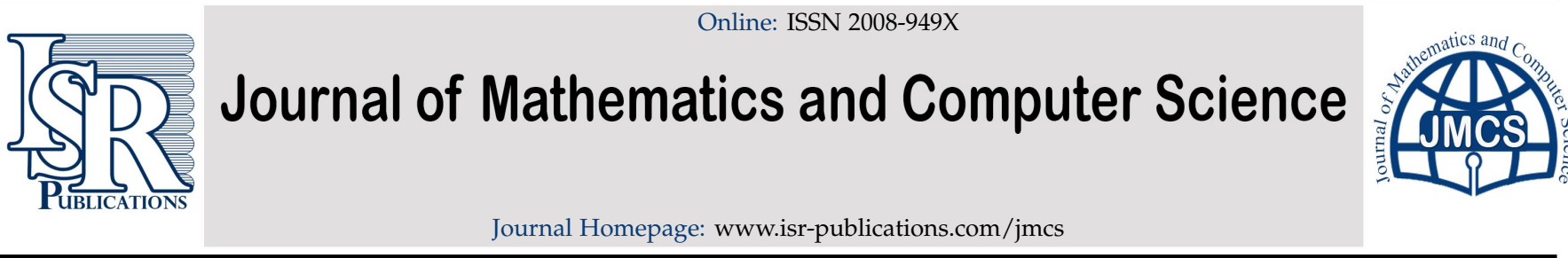

\title{
Weighted Jessen's functionals and exponential convexity
}

Rishi Naeem*, Matloob Anwar

School of Natural Sciences, National University of Sciences and Technology, Islamabad, Pakistan.

\begin{abstract}
In this paper, we give a refinement of the well known Jessen's inequality via weight functions. We discuss m-exponential convexity of the functions associated with these weighted Jessen's functionals. Cauchy and Lagrange mean value theorems are also given that are useful in the construction of means with Stolarsky property.
\end{abstract}

Keywords: Convex function, Jessen's inequality, log-convex functions, exponentially convex function, mean value theorems, Stolarsky means.

2010 MSC: 26A24, 26A48, 26A51, 26D15.

(C)2019 All rights reserved.

\section{Introduction and preliminaries}

A real valued function $\Psi$ on $S$, where $S$ is a convex set, is called convex if epigraph of $\Psi$ is a convex set. Alternatively, a function $\Psi$ is convex if

$$
\Psi(\rho x+(1-\rho) y) \leqslant \rho \Psi(x)+(1-\rho) \Psi(y)
$$

for all $x, y \in S$ and $\rho \in[0,1]$.

The well known king of inequalities, i.e., the Jensen's inequality is the generalization of above inequality (see $[3,11]$ ). The Jensen's inequality states that if $S \subseteq \mathbb{R}$ and $\Psi: S \rightarrow \mathbb{R}$ is convex then for all $x_{i} \in S,(1 \leqslant i \leqslant n)$ and $\rho_{i}(1 \leqslant i \leqslant n)$ non negative real such that $\rho_{1}+\cdots+\rho_{\mathfrak{n}}=1$ we have

$$
\Psi\left(\sum_{i=1}^{n} \rho_{i} x_{i}\right) \leqslant \sum_{i=1}^{n} \rho_{i} \Psi\left(x_{i}\right) .
$$

The classical integral form of Jensen's inequality states that if $\lambda$ is a positive measure on a $\sigma$-algebra $\mathcal{A}$ on a set $X$, so that $\lambda(X)=1$. If $h \in \mathrm{L}^{1}(\lambda), h(x) \in S \subseteq \mathbb{R}$ for all $x \in X$ and $\Psi: S \rightarrow \mathbb{R}$ is convex (see for example $[15,18])$, then

$$
\Psi\left(\int_{X} h \mathrm{~d} \lambda\right) \leqslant \int_{X}(\Psi \circ h) \mathrm{d} \lambda
$$

\footnotetext{
${ }^{*}$ Corresponding author

Email addresses: rishi.naeem@sns.nust.edu.pk (Rishi Naeem), manwar@sns.nust.edu.pk (Matloob Anwar)

doi: $10.22436 /$ jmcs.019.03.04
}

Received: 2019-03-22 Revised: 2019-04-25 Accepted: 2019-04-30 
Let $\left(X, \mathcal{A}, \lambda_{1}\right)$ and $\left(Y, \mathcal{B}, \lambda_{2}\right)$ are two probability measure spaces. A (separately) weight function $\omega$ is defined as a product-measurable mapping $\omega: X \times Y \rightarrow[0, \infty)$, see for example [18], such that

$$
\int_{X} \omega(x, y) d \lambda_{1}(x)=1(\text { for every } y \in Y), \quad \int_{Y} \omega(x, y) d \lambda_{2}(y)=1(\text { for every } x \in X) .
$$

The following result is a refined form of integral Jensen's inequality via weight function.

Theorem $1.1([16])$. Suppose that $\left(X, \mathcal{A}, \lambda_{1}\right)$ and $\left(Y, \mathcal{B}, \lambda_{2}\right)$ are two probability measure spaces and $\omega$ is a weight function (defined in (1.1)). If $S \subseteq \mathbb{R}, h \in \mathrm{L}^{1}\left(\lambda_{1}\right), h(x) \in S$ for all $x \in X$ and $\Psi: S \rightarrow \mathbb{R}$ is a real convex function, then

$$
\int_{Y} \Psi\left(\int_{X} h(x) \omega(x, y) d \lambda_{1}(x)\right) d \lambda_{2}(y)
$$

has meaning and

$$
\Psi\left(\int_{X} h d \lambda_{1}\right) \leqslant \int_{Y} \Psi\left(\int_{X} h(x) \omega(x, y) d \lambda_{1}(x)\right) d \lambda_{2}(y) \leqslant \int_{X}(\Psi \circ h) d \lambda_{1} .
$$

Let $\mathrm{E}(\neq \emptyset)$ and $\mathrm{L}$ be a linear class of real valued functions $h: E \rightarrow \mathbb{R}$ having the properties:

$\mathrm{L}_{1}: h, k \in \mathrm{L} \Rightarrow(\alpha \mathrm{h}+\beta \mathrm{k}) \in \mathrm{L}$ for all $\alpha, \beta \in \mathbb{R}$;

$\mathrm{L}_{2}: 1 \in \mathrm{L}$ that is if $\mathrm{h}(\mathrm{l})=1$ for $l \in \mathrm{E}$, then $h \in \mathrm{L}$.

We also consider positive linear functionals $A: L \rightarrow \mathbb{R}$ possess the properties:

$$
A_{1}: A(\alpha h+\beta k)=\alpha A(h)+\beta A(k) \text { for } h, k \in L, \alpha, \beta \in \mathbb{R} \text {; }
$$

$A_{2}: h \in L, h(l) \geqslant 0$ on $E \Rightarrow A(h) \geqslant 0$ ( $A$ is positive).

The mapping $A$ is said to be normalized if

$$
A_{3}: A(1)=1 \text {. }
$$

By a weight function, we mean a mapping $\omega: E \times E \rightarrow \mathbb{R}^{+}$such that

$$
A(\omega(x, y))=1(\text { for each } y \text { in } E), \quad B(\omega(x, y))=1(\text { for each } x \text { in } E),
$$

where $A$ and $B$ satisfy the properties $A_{1}, A_{2}$, and $A_{3}$.

Jessen in [7] (see also [15]) gave the following generalization of Jensen's inequality for positive linear functionals.

Theorem 1.2. Let $\mathrm{L}$ satisfy properties $\mathrm{L}_{1}, \mathrm{~L}_{2}$ on a nonempty set $\mathrm{E}$ and let $\Psi: S \rightarrow \mathbb{R}$ be a continuous convex function on an interval $S \subseteq \mathbb{R}$. If $A$ is a positive linear functional on $\mathrm{L}$ with $\mathrm{A}(1)=1$, then for all $\mathrm{h} \in \mathrm{L}$ such that $\Psi(\mathrm{h}) \in \mathrm{L}$ we have $\mathrm{A}(\mathrm{h}) \in \mathrm{S}$ and

$$
\Psi(A(h)) \leqslant A(\Psi(h)) .
$$

The concept of m-exponentially convex functions was introduced by Pečarić and Perić in [14]. An elegant method from [6] is used to construct m-exponential convexity, see [1, 2, 4, 5, 8-10, 12, 13].

The next section deals with the investigation of m-exponential convexity of the kind of functionals that are related with the refined form of weighted integral Jensen's inequality (1.2). The result are deduced about exponential convexity as well as the log-convexity. Lagrange and Cauchy type mean value theorems are established and the construction of means is made using Stolarsky property. In Section 3, we refine the Jessen's inequality via weight function and discuss m-exponential convexity of the functions associated with these linear functionals. We present several families of functions related to our main results and mean value theorems are also given. On the other hand, in [13] we proved the counterpart of this refined inequality over a compact interval $S=[\eta, \zeta]$ and investigate its exponential and log-convexity. 


\section{2. m-exponential convexity of weighted integral Jensen's inequality}

A real valued function $\Psi: S \rightarrow \mathbb{R}$ is m-exponentially convex if it is m-exponentially $\mathcal{J}$-convex and continuous on I; hence it is an exponentially convex function (for detail see [14]).

Remark 2.1. A positive real valued function $\Psi: S \rightarrow \mathbb{R}$ is log-J-convex if and only if it is 2-exponentially $\mathcal{J}$-convex. Converse is true provided that $\Psi$ is continuous also.

Remark 2.2. If the divided difference $\left[s_{1}, s_{2} ; \Psi\right] \geqslant 0$ for every $s_{1}, s_{2} \in S$, then $\Psi$ is increasing on its domain.

Lemma 2.3 ([5]). If $\Psi: S \rightarrow \mathbb{R}$ is log-convex, then for $l<r<s(l, r, s \in S)$,

$$
(\Psi(r))^{s-l} \leqslant(\Psi(l))^{s-r}(\Psi(s))^{r-l} .
$$

Remark 2.4. We consider the following functionals under the assumption of Theorem 1.1,

$$
\begin{aligned}
& \Omega(\Psi)=\int_{Y} \Psi\left(\int_{X} h(x) \omega(x, y) d \lambda_{1}(x)\right) d \lambda_{2}(y)-\Psi\left(\int_{X} h d \lambda_{1}\right), \\
& \digamma(\Psi)=\int_{X}(\Psi \circ h) d \lambda_{1}-\int_{Y} \Psi\left(\int_{X} h(x) \omega(x, y) d \lambda_{1}(x)\right) d \lambda_{2}(y) .
\end{aligned}
$$

Then $\Omega(\Psi)$ and $\digamma(\Psi)$ are positive.

We construct m-exponentially convex functions and exponentially convex functions by applying an elegant method from [6]. In the following theorem and its corollaries we have used the notation as: $\mathcal{C}$ is a real open interval, $S$ is any real interval, and $\Gamma=\left\{g_{l} \mid l \in \mathcal{C}\right\}$ is a family of functions defined on $S . \Omega(\Psi)$ is as given in (2.1). Then we have the following useful results which produce new m-exponentially convex functions.

Theorem 2.5. Suppose that the function $l \mapsto\left[s_{1}, s_{2}, s_{3} ; g_{l}\right]$ is m-exponentially $\mathcal{J}$-convex on $\mathcal{C}$, where $s_{1}, s_{2}, s_{3}$ are distinct points of $\mathrm{S}$. Then $\mathrm{l} \mapsto \Omega\left(\mathrm{g}_{\mathrm{l}}\right)$ is an m-exponentially $\mathcal{J}$-convex function on $\mathrm{C}$. In addition, the continuity of this function implies the m-exponential convexity on $\mathrm{C}$.

Proof. Assume $l_{i}, l_{j} \in \mathcal{C}, l_{i j}=\frac{l_{i}+l_{j}}{2}$ and $a_{i}, a_{j} \in \mathbb{R}$ for $i, j \in\{1, \ldots, m\}$. Consider the function $\Delta$ on $S$ defined as

$$
\Delta(s)=\sum_{i, j=1}^{m} a_{i} a_{j} g_{l_{i j}}(s) .
$$

Being the linear combination of continuous functions, $\Delta$ is continuous. The hypothesis of m-exponential J-convexity of $l \mapsto\left[s_{1}, s_{2}, s_{3} ; g_{l}\right]$ yields

$$
\left[s_{1}, s_{2}, s_{3} ; \Delta\right]=\sum_{i, j=1}^{m} a_{i} a_{j}\left[s_{1}, s_{2}, s_{3} ; g_{l_{i j}}\right] \geqslant 0,
$$

implying the convexity of $\Delta$ on $S$. Hence $\Omega(\Delta) \geqslant 0$. Further the linearity of $\Omega$, gives

$$
\sum_{i, j=1}^{m} a_{i} a_{j} \Omega\left(g_{l_{i j}}\right) \geqslant 0 .
$$

We conclude that the function $l \mapsto \Omega\left(g_{l}\right)$ is an m-exponentially $\mathcal{J}$-convex function on $\mathcal{C}$.

The following corollaries are consequences of above theorem.

Corollary 2.6. Suppose that the function $\mathrm{l} \mapsto\left[\mathrm{s}_{1}, \mathrm{~s}_{2}, \mathrm{~s}_{3} ; \mathrm{g}_{\mathrm{l}}\right]$ is exponentially $\mathcal{J}$-convex on $\mathcal{C}$, where $\mathrm{s}_{1}, \mathrm{~s}_{2}, \mathrm{~s}_{3}$ are distinct points of $\mathrm{S}$. Then $\mathrm{l} \mapsto \Omega\left(\mathrm{g}_{\mathrm{l}}\right)$ is an exponentially J-convex function on $\mathrm{C}$. In addition, the continuity of this function implies the exponential convexity on $\mathrm{C}$. 
Corollary 2.7. Suppose that the function $\mathrm{l} \mapsto\left[\mathrm{s}_{1}, \mathrm{~s}_{2}, \mathrm{~s}_{3} ; \mathrm{g}_{\mathrm{l}}\right]$ is 2-exponentially $\mathcal{J}$-convex on $\mathcal{C}$, where $\mathrm{s}_{1}, \mathrm{~s}_{2}, \mathrm{~s}_{3}$ are distinct points of $\mathrm{S}$. Then the following statements hold.

(i) The continuity of the function $l \mapsto \Omega\left(g_{l}\right)$ implies the 2-exponential convexity of $l \mapsto \Omega\left(g_{l}\right)$ on $\mathcal{C}$, and hence the function is log-convex. That is for $l, r, s \in \mathcal{C}$ such that $\mathrm{r}<\mathrm{s}<\mathrm{l}$, the following holds

$$
\Omega^{l-r}\left(g_{s}\right) \leqslant \Omega^{l-s}\left(g_{r}\right) \Omega^{s-r}\left(g_{l}\right) .
$$

(ii) Assume that the function $\mathrm{l} \mapsto \Omega\left(\mathrm{g}_{\mathrm{l}}\right)$ on $\mathrm{C}$ is strictly positive and differentiable. Then for $\mathrm{l} \leqslant \mathrm{u}$ and $\mathrm{r} \leqslant v$, $(\mathrm{l}, \mathrm{r}, \mathrm{u}, v \in \mathcal{C})$ yields

$$
\tau(l, r, \Omega) \leqslant \tau(u, v ; \Omega),
$$

where

$$
\tau(l, r ; \Omega)= \begin{cases}\left(\frac{\Omega\left(g_{l}\right)}{\Omega\left(g_{r}\right)}\right)^{\frac{1}{l-r}}, & l \neq r, \\ \exp \left(\frac{\frac{d}{d l}\left(\Omega\left(g_{l}\right)\right)}{\Omega\left(g_{l}\right)}\right), & \text { otherwise. }\end{cases}
$$

Proof.

(i) This is an immediate consequence of Theorem 2.5 and Remark 2.1.

(ii) The log-convexity of $l \mapsto \Omega\left(g_{l}\right)$ on $\mathcal{C}$ follows from (i). It implies the convexity of $l \mapsto \log \Omega\left(g_{l}\right)$ on $\mathcal{C}$. Applying [14, Proposition 3.2] with $l \leqslant u, r \leqslant v$, we get

$$
\frac{\log \Omega\left(g_{l}\right)-\log \Omega\left(g_{r}\right)}{l-r} \leqslant \frac{\log \Omega\left(g_{u}\right)-\log \Omega\left(g_{v}\right)}{u-v},
$$

thus we have

$$
\tau(l, r, \Omega) \leqslant \tau(u, v ; \Omega) .
$$

The cases $l=r$ and $u=v$ follow from (2.3) as limit cases.

Now we present different families of functions to investigate exponential convexity. The following lemma will be useful to construct new exponentially convex functions. Since the below mentioned result is the simple consequence of some basic examples and remarks given in [6], so we omit the proof.

\section{Lemma 2.8 .}

(i) Define a real valued function $f_{l}$ on $S=\mathbb{R}$ by $f_{l}(s)=\frac{1}{l^{2}} \exp (l s),(l>0)$. This implies the exponential convexity on $(0, \infty)$ of $\mathrm{l} \mapsto \frac{\mathrm{d}^{2}}{\mathrm{ds}^{2}} \mathrm{f}_{\mathrm{l}}(\mathrm{s})$ for each $\mathrm{s} \in \mathrm{S}$.

(ii) Let $\mathrm{g}_{\mathrm{l}}$ be a real valued function defined on $\mathrm{S}=\mathbb{R}^{+}$by $\mathrm{g}_{\mathrm{l}}(\mathrm{s})=\frac{\mathrm{s}^{\mathrm{l}}}{\mathrm{l}(\mathrm{l}-1)},(\mathrm{l}>1)$. It implies the exponential convexity on $(1, \infty)$ of the function $\mathrm{l} \mapsto \frac{\mathrm{d}^{2}}{\mathrm{ds}^{2}} \mathrm{~g}_{\mathrm{l}}(\mathrm{s})$ for each $\mathrm{s} \in \mathrm{S}$.

(iii) Consider a positive real valued function defined on $S=\mathbb{R}^{+}$by $h_{l}(s)=\frac{l^{-s}}{(\log l)^{2}},(l>1)$. It implies the exponential convexity on $(1, \infty)$ of the function $\mathrm{l} \mapsto \frac{\mathrm{d}^{2}}{\mathrm{ds}^{2}} \mathrm{~h}_{\mathrm{l}}(\mathrm{s})$ for each $\mathrm{s} \in \mathrm{S}$.

(iv) Define a positive real valued function $k_{l}$ on $S=\mathbb{R}^{+}$by $k_{l}(s)=\frac{1}{l} \exp (-s \sqrt{l}),(l>0)$. This implies the exponential convexity on $(0, \infty)$ of the function $\mathrm{l} \mapsto \frac{\mathrm{d}^{2}}{\mathrm{ds}^{2}} \mathrm{k}_{\mathrm{l}}(\mathrm{s})$ for each $\mathrm{s} \in \mathrm{S}$.

Remark 2.9. The following positive functionals are useful in defining the basic inequality of log-convex functions.

$$
\begin{aligned}
& \Omega\left(f_{l}\right)=\frac{1}{l^{2}}\left(\int_{Y} \exp \left(l \int_{X} h(x) \omega(x, y) d \lambda_{1}(x)\right) d \lambda_{2}(y)-\exp \left(l \int_{X} h d \lambda_{1}\right)\right), \\
& \Omega\left(g_{l}\right)=\frac{1}{l(l-1)}\left(\int_{Y}\left(\int_{X} h(x) \omega(x, y) d \lambda_{1}(x)\right)^{l} d \lambda_{2}(y)-\left(\int_{X} h d \lambda_{1}\right)^{l}\right), \\
& \Omega\left(h_{l}\right)=\frac{1}{(\log l)^{2}}\left(\int_{Y} l^{-\left(\int_{X} h(x) \omega(x, y) d \lambda_{1}(x)\right)} d \lambda_{2}(y)-l-\left(\int_{X} h d \lambda_{1}\right)\right), \\
& \Omega\left(k_{l}\right)=\frac{1}{l}\left(\int_{Y} \exp \left(-\sqrt{l} \int_{X} h(x) \omega(x, y) d \lambda_{1}(x)\right) d \lambda_{2}(y)-\exp \left(-\sqrt{l} \int_{X} h d \lambda_{1}\right)\right) .
\end{aligned}
$$


Theorem 2.10. Let $\Omega(\Psi)$ be the linear functional defined by (2.1) and consider the function $\phi_{i}:(0, \infty) \rightarrow \mathbb{R}$ for $i=1,4$ and $\phi_{i}:(1, \infty) \rightarrow \mathbb{R}$ for $i=2,3$ defined as $\phi_{1}(l)=\Omega\left(f_{l}\right), \phi_{2}(l)=\Omega\left(g_{l}\right), \phi_{3}(l)=\Omega\left(h_{l}\right), \phi_{4}(l)=$ $\Omega\left(k_{l}\right)$, where $f_{l}, g_{l}, h_{l}$ and $k_{l}$ are defined in Lemma 2.8. Then

(i) the functions $\phi_{i}$ are continuous on $(0, \infty)$ for $i=1,4$ and continuous on $(1, \infty)$ for $i=2,3$;

(ii) if $m \in \mathbb{N}, l_{1}, \ldots, l_{m} \in(0, \infty)$ for $i=1,4$ and $l_{1}, \ldots, l_{m} \in(1, \infty)$ for $i=2,3$. Then $\left[\phi_{i}\left(\frac{l_{j}+l_{k}}{2}\right)\right]_{j, k=1}^{m}$ are positive semidefinite matrices;

(iii) $\phi_{i}$ are exponentially convex on $(0, \infty)$ when $i=1,4$ and on $(1, \infty)$ for $i=2,3$;

(iv) let $l, r, s \in(0, \infty)$ for $i=1,4$ and $r, s, l \in(1, \infty)$ for $i=2,3$ with $l<r<s$, then

$$
\left(\phi_{i}(r)\right)^{s-l} \leqslant\left(\phi_{i}(l)\right)^{s-r}\left(\phi_{i}(s)\right)^{r-l},
$$

where $\phi_{i}(l)$ for $i=1,2,3,4$ are defined in Remark 2.9;

(v) if the functions $\phi_{i}$ are differentiable and strictly positive on $(0, \infty)$ for $i=1,4$ and on $(1, \infty)$ for $i=2,3$, then for every $l, r, u, v \in(0, \infty)$ for $i=1,4$ and $l, r, u, v \in(1, \infty)$ for $i=2,3$ such that $l \leqslant u$ and $r \leqslant v$, we have

$$
\tau\left(l, r, \phi_{i}\right) \leqslant \tau\left(u, v ; \phi_{i}\right)
$$

where

$$
\tau\left(l, r ; \phi_{i}\right)= \begin{cases}\left(\frac{\left.\phi_{i}(l)\right)}{\phi_{i}(r)}\right)^{\frac{1}{l-r}}, & l \neq r \\ \exp \left(\frac{\frac{d}{d l}\left(\phi_{i}(l)\right)}{\phi_{i}(l)}\right), & \text { otherwise. }\end{cases}
$$

Proof.

(i) The continuity of these functions is obvious.

(ii) Let $d_{j}, l_{j} \in \mathbb{R}$, where $j=1, \ldots, m$ ( $m$ is any natural number). Define the auxiliary function $\Delta_{1}$ on $\mathrm{S}=\mathbb{R}$ by

$$
\Delta_{1}(s)=\sum_{j, k=1}^{m} d_{j} d_{k} f_{\frac{l_{j}+l_{k}}{2}}(s) .
$$

Since

$$
\Delta_{1}^{\prime \prime}(s)=\sum_{j, k=1}^{m} d_{j} d_{k} \frac{d^{2}}{d s^{2}} f_{\frac{l_{j}+l_{k}}{2}}(s) \geqslant 0
$$

for $s \in S$ by Lemma 2.8. This implies $\Delta_{1}$ is convex. Now Theorem 1.1 implies that $\Omega\left(\Delta_{1}\right) \geqslant 0$. This means that $\left[\phi_{1}\left(\frac{l_{j}+l_{k}}{2}\right)\right]_{j, k=1}^{m}$ is a positive semidefinite matrix.

To prove the remaining positive semidefinite matrices, we can define the auxiliary functions $\Delta_{i}$ for $i=2,3,4$ in the similar manner.

(iii) and (iv) are simple consequences of (i), (ii), and Lemma 2.3. We can easily prove (v) by using basic inequality of log-convex functions given in part (iv).

\subsection{Mean value theorems}

To prove our main results, the following lemma is very useful.

Lemma 2.11 ([15]). Suppose $\Psi \in C^{2}([\mu, v])$ and $\Psi:[\mu, v] \rightarrow \mathbb{R}$, where $[\mu, v] \subseteq \mathbb{R}, \Psi^{\prime \prime}$ is bounded. Assume $\mathfrak{d}=\inf _{l \in[\mu, v]} \Psi^{\prime \prime}(l), \mathfrak{D}=\sup _{\mathfrak{l} \in[\mu, v]} \Psi^{\prime \prime}(l)$. Then the functions $\Psi_{1}, \Psi_{2}:[\mu, v] \rightarrow \mathbb{R}$ defined by

$$
\Psi_{1}(l)=\frac{\mathfrak{D}}{2} l^{2}-\Psi(l), \quad \Psi_{2}(l)=\Psi(l)-\frac{\mathfrak{d}}{2} l^{2}
$$

are convex. 
Theorem 2.12. Assume $\Psi: S=[\mu, v] \rightarrow \mathbb{R}, \Psi \in C^{2}(S)$, where $S \subseteq \mathbb{R}$ is compact. If $\left(X, \mathcal{A}, \lambda_{1}\right)$ and $\left(Y, \mathcal{B}, \lambda_{2}\right)$ are two probability measure spaces and $\omega$ is a weight function (defined in (1.1)) such that $\mathrm{h} \in \mathrm{L}^{1}\left(\lambda_{1}\right), \mathrm{h}(\mathrm{X}) \in \mathrm{S}$ for all $x \in X$. Then there exists $\varpi \in S$ such that

$$
\int_{Y} \Psi\left(\int_{X} h(x) \omega(x, y) d \lambda_{1}(x)\right) d \lambda_{2}(y)-\Psi\left(\int_{X} h d \lambda_{1}\right)=\zeta \Psi^{\prime \prime}(\varpi)
$$

where

$$
\zeta=\frac{1}{2}\left[\int_{Y}\left(\int_{X} h(x) \omega(x, y) d \lambda_{1}(x)\right)^{2} d \lambda_{2}(y)-\left(\int_{X} h d \lambda_{1}\right)^{2}\right] .
$$

Proof. Suppose $\mathfrak{D}=\max _{l \in S} \Psi^{\prime \prime}(l)$ and $\mathfrak{d}=\min _{l \in S} \Psi^{\prime \prime}(l)$. Then the convexity of the functions $\Psi_{1}, \Psi_{2}: S \rightarrow$ $\mathbb{R}$ follows from Lemma 2.11. Since they are also continuous. Applying left-hand side of the inequality (1.2), we get

$$
\int_{Y} \Psi\left(\int_{X} h(x) \omega(x, y) d \lambda_{1}(x)\right) d \lambda_{2}(y)-\Psi\left(\int_{X} h d \lambda_{1}\right) \leqslant \zeta \mathfrak{D}
$$

and

$$
\int_{Y} \Psi\left(\int_{X} h(x) \omega(x, y) d \lambda_{1}(x)\right) d \lambda_{2}(y)-\Psi\left(\int_{X} h d \lambda_{1}\right) \geqslant \zeta \mathfrak{d} .
$$

Now combining these two inequalities and since $\psi^{\prime \prime}$ is continuous, there exists $\varpi \in S\left(\mathfrak{d} \leqslant \Psi^{\prime \prime}(\varpi) \leqslant \mathfrak{D}\right)$ such that (2.4) holds.

Theorem 2.13. Assume $\Phi, \Psi: S=[\mu, v] \rightarrow \mathbb{R}, \Phi, \Psi \in C^{2}(S)$, where $S \subseteq \mathbb{R}$ is compact. If $\left(X, \mathcal{A}, \lambda_{1}\right)$ and $\left(\mathrm{Y}, \mathcal{B}, \lambda_{2}\right)$ are two probability measure spaces and $\omega$ is a weight function (defined in (1.1)) such that $\mathrm{h} \in \mathrm{L}^{1}\left(\lambda_{1}\right), \mathrm{h}(\mathrm{x}) \in \mathrm{S}$ for all $x \in X$. Then $\varpi \in S$ exists that ensures

$$
\begin{aligned}
\Psi^{\prime \prime}(\varpi) & {\left[\int_{Y} \Phi\left(\int_{X} h(x) \omega(x, y) d \lambda_{1}(x)\right) d \lambda_{2}(y)-\Phi\left(\int_{X} h d \lambda_{1}\right)\right] } \\
= & \Phi^{\prime \prime}(\varpi)\left[\int_{Y} \Psi\left(\int_{X} h(x) \omega(x, y) d \lambda_{1}(x)\right) d \lambda_{2}(y)-\Psi\left(\int_{X} h d \lambda_{1}\right)\right] .
\end{aligned}
$$

Proof. Suppose a function $k \in C^{2}(S)$ defined as $k=c_{1} \Phi-c_{2} \Psi$, where $c_{1}$ and $c_{2}$ are defined by

$$
c_{1}=\int_{Y} \Psi\left(\int_{X} h(x) \omega(x, y) d \lambda_{1}(x)\right) d \lambda_{2}(y)-\Psi\left(\int_{X} h d \lambda_{1}\right)
$$

and

$$
c_{2}=\int_{Y} \Phi\left(\int_{X} h(x) \omega(x, y) d \lambda_{1}(x)\right) d \lambda_{2}(y)-\Phi\left(\int_{X} h d \lambda_{1}\right)
$$

As $k \in C^{2}(S)$, applying Theorem 2.12 on $k$ ensures that there exists some $\varpi \in S$ such that the following holds

$$
\int_{Y} k\left(\int_{X} h(x) \omega(x, y) d \lambda_{1}(x)\right) d \lambda_{2}(y)-k\left(\int_{X} h d \lambda_{1}\right)=\zeta k^{\prime \prime}(\varpi) .
$$

The left-hand side of this equation equals to zero and the term $\zeta$ on the right-hand side is non zero, so we have that $k^{\prime \prime}(\varpi)=0$. Thus the assertion of our theorem follows directly.

Remark 2.14. If $\Phi^{\prime \prime} / \Psi^{\prime \prime}$ is invertible, then means of various kinds can be defined by (2.5). That is,

$$
\varpi=\left(\frac{\Phi^{\prime \prime}}{\Psi^{\prime \prime}}\right)^{-1}\left(\frac{\Omega(\Phi)}{\Omega(\Psi)}\right)
$$


If we apply Cauchy type mean value Theorem 2.13 on functions $\Phi=f_{l}$ and $\Psi=f_{r}$ (defined in Lemma 2.8), it yields

$$
M(l, r ; \Omega)=\log \tau(l, r ; \Omega)
$$

satisfy

$$
\mu \leqslant M(l, r ; \Omega) \leqslant v,
$$

where

$$
\tau(l, r ; \Omega)= \begin{cases}\left(\frac{\Omega\left(f_{l}\right)}{\Omega\left(f_{r}\right)}\right)^{\frac{1}{l-r}}, & l \neq r, \\ \exp \left(\frac{\Omega\left(i d . f_{l}\right)}{\Omega\left(f_{l}\right)}\right) \cdot \exp \left(-\frac{2}{l}\right), & l=r \neq 0 .\end{cases}
$$

If we set $\mu=\min _{l \in[\mu, v]}\{f(l)\}$ and $v=\max _{l \in[\mu, v]}\{f(l)\}$, then

$$
\min _{l \in[\mu, v]}\{f(l)\} \leqslant M(l, r ; \Omega) \leqslant \max _{l \in[\mu, v]}\{f(l)\}
$$

which shows that $M(l, r ; \Omega)$ are means of $f(l), l \in[\mu, v]$. If $l \leqslant u, r \leqslant v$ where $l, r, u, v \in \mathbb{R}$, then it yields by Theorem 2.10 that these means are monotonic.

Now, if we apply Cauchy type mean value Theorem 2.13 on functions $\Phi=g_{l}$ and $\Psi=g_{r}$ (defined in Lemma 2.8), this yields that there exists $\varpi \in[\mu, v]$ such that

$$
\varpi^{l-r}=\frac{\Omega\left(g_{l}\right)}{\Omega\left(g_{r}\right)} .
$$

Since the function $\varpi \mapsto \varpi^{l-r}$ is invertible for $l \neq r$, we get

$$
\mu \leqslant\left(\frac{\Omega\left(g_{l}\right)}{\Omega\left(g_{r}\right)}\right)^{\frac{1}{l-r}} \leqslant v .
$$

If we set $\mu=\min _{l \in[\mu, v]}\{f(l)\}$ and $v=\max _{l \in[\mu, v]}\{f(l)\}$, then in this case $\tau(l, r ; \Omega)$ are means of $f(l), l \in$ $[\mu, v]$, where

$$
\tau(l, r ; \Omega)= \begin{cases}\left(\frac{\Omega\left(g_{l}\right)}{\Omega\left(g_{r}\right)}\right)^{\frac{1}{l-r}}, & l \neq r, \\ \exp \left(\frac{1-2 l}{l(l-1)}\right) \cdot \exp \left(-\frac{\Omega\left(g_{0} g_{l}\right)}{\Omega\left(g_{l}\right)}\right), & l=r \neq 1 .\end{cases}
$$

Note that in this case, the monotonicity property also holds for $\tau(l, r ; \Omega)$.

Remark 2.15. We can construct similar results for the positive functional $\digamma(\Psi)$ defined in (2.2). Moreover, by introducing suitable weight functions, Rooin gave the refinement of the discrete Jensen's inequality and some of its applications (see [17]). We can prove similar results for the positive functionals associated with this refined form of discrete Jensen's inequality.

\section{A new refinement of Jessen's inequality and exponential convexity}

The following theorem is the refinement of Jessen's inequality.

Theorem 3.1. Let $\mathrm{L}$ satisfies properties $\mathrm{L}_{1}$ and $\mathrm{L}_{2}$ on a nonempty set $\mathrm{E}$, and assume that $\Psi$ is a continuous convex function on an interval $\mathrm{S} \subseteq \mathbb{R}$. If $\mathrm{A}$ and $\mathrm{B}$ are positive linear functionals with $\mathrm{A}(1)=\mathrm{B}(1)=1$ and $\omega$ is a weight function (defined in (1.3)) then for all $h, h \cdot \omega \in L$ such that $\Psi(A(h \cdot \omega)), \Psi(h) \in L$ we have $A(h \cdot \omega), A(h) \in S$ and

$$
\Psi(A(h)) \leqslant B(\Psi(A(h \cdot \omega))) \leqslant A(\Psi(h)) .
$$


Proof.

$$
B(\Psi(A(h \cdot \omega))) \geqslant \Psi(B(A(h \cdot \omega))) \geqslant \Psi(B(A(h)))=\Psi(A(h)) .
$$

Let $S=[a, b]$. From $a \leqslant h(l) \leqslant b$ for all $l \in E$ we obtain $a \leqslant A(h) \leqslant b$ and $a \leqslant A(h \cdot \omega) \leqslant b$. For arbitrary but fixed $\varepsilon>0$ there exist $\alpha, \beta \in \mathbb{R}$ such that for $\rho=\alpha \rho_{0}+\beta \rho_{1}\left(\rho_{j}(l)=l^{j}\right.$ for $\left.j=0,1\right)$ we have (i) $\rho \leqslant \Psi$, it yields $\rho(A(h)) \leqslant \Psi(A(h \cdot \omega))$. (ii) $\rho(A(h)) \geqslant \Psi(A(h \cdot \omega))-\varepsilon$ which implies $B(\rho(A(h))) \geqslant$ $B(\Psi(A(h \cdot \omega)))-\varepsilon$. (If $a<A(h)<b$ and $a<A(h \cdot \omega)<b$ or if $h, h \cdot \omega$ have finite derivatives in $[a, b]$, we can replace (ii) by $\rho(A(h))=\Psi(A(h \cdot \omega)))$. Now (i) implies $\rho \circ h \leqslant \Psi \circ h$; hence

$$
A(\Psi \circ h) \geqslant A(\rho \circ h)=\alpha+\beta A(h)=B(\rho(A(h))) \geqslant B(\Psi(A(h \cdot \omega)))-\varepsilon .
$$

Since $\varepsilon$ is arbitrary, the proof is complete.

If $\omega=1$, then we deduce the following result.

Corollary 3.2. Let $\mathrm{L}$ satisfies properties $\mathrm{L}_{1}$ and $\mathrm{L}_{2}$ on a nonempty set $\mathrm{E}$, and assume that $\Psi$ is a continuous convex function on an interval $S \subseteq \mathbb{R}$. If $\mathrm{A}$ and $\mathrm{B}$ are positive linear functionals with $\mathrm{A}(1)=\mathrm{B}(1)=1$, then for all $\mathrm{h} \in \mathrm{L}$ such that $\Psi(\mathrm{A}(\mathrm{h})), \Psi(\mathrm{h}) \in \mathrm{L}$ we have $\mathrm{A}(\mathrm{h}) \in \mathrm{S}$ and

$$
\Psi(A(h)) \leqslant B(\Psi(A(h))) \leqslant A(\Psi(h)) .
$$

Remark 3.3. We consider the following functionals under the assumption of Theorem 3.1,

$$
\begin{aligned}
& \Lambda(\Psi)=B(\Psi(A(h \cdot \omega)))-\Psi(A(h)), \\
& \neg(\Psi)=A(\Psi(h))-B(\Psi(A(h \cdot \omega))) .
\end{aligned}
$$

Then $\Lambda(\Psi)$ and $\urcorner(\Psi)$ are positive.

In the following theorem and its corollaries we have used the notations as: $\mathcal{C}$ is a real open interval, $S$ is any interval, and $\Theta=\left\{g_{l} \mid l \in \mathcal{C}\right\}$ is a family of functions defined on $S . \Lambda(\Psi)$ is as given in Remark 3.3. Then the following results produce new m-exponentially convex functions. Since the proofs are analogous to those in the continuous case given in the previous section, so we omit the proofs.

Theorem 3.4. Suppose the function $\mathrm{l} \mapsto\left[\mathrm{s}_{1}, \mathrm{~s}_{2}, \mathrm{~s}_{3} ; \mathrm{g}_{\mathrm{l}}\right]$ is m-exponentially $\mathcal{J}$-convex on $\mathcal{C}$, where $s_{1}, \mathrm{~s}_{2}, \mathrm{~s}_{3}$ are distinct points of $\mathrm{S}$. Then $\mathrm{l} \mapsto \Lambda\left(\mathrm{g}_{\mathrm{l}}\right)$ is an m-exponentially J-convex function on $\mathrm{C}$. In addition, the continuity of this function implies m-exponential convexity on $\mathrm{C}$.

The following corollaries are consequences of above theorem.

Corollary 3.5. Suppose the function $\mathrm{l} \mapsto\left[\mathrm{s}_{1}, \mathrm{~s}_{2}, \mathrm{~s}_{3} ; \mathrm{g}_{\mathrm{l}}\right]$ is exponentially $\mathcal{J}$-convex on $\mathcal{C}$, where $\mathrm{s}_{1}, \mathrm{~s}_{2}, \mathrm{~s}_{3}$ are distinct points of $\mathrm{S}$. Then $\mathrm{l} \mapsto \Lambda\left(\mathrm{g}_{\mathrm{l}}\right)$ is an exponentially $\mathcal{J}$-convex function on $\mathrm{C}$. In addition, the continuity of this function implies the exponential convexity on $\mathrm{C}$.

Corollary 3.6. Suppose the function $\mathrm{l} \mapsto\left[\mathrm{s}_{1}, \mathrm{~s}_{2}, \mathrm{~s}_{3} ; \mathrm{g}_{\mathrm{l}}\right]$ is 2-exponentially $\mathcal{J}$-convex on $\mathrm{C}$, where $\mathrm{s}_{1}, \mathrm{~s}_{2}, \mathrm{~s}_{3}$ are distinct points of $\mathrm{S}$. Then $\mathrm{l} \mapsto \Lambda\left(\mathrm{g}_{\mathrm{l}}\right)$ is 2-exponentially $\mathrm{J}$-convex function on $\mathrm{C}$. In addition, the continuity of this function implies the exponential convexity on $\mathcal{C}$ and thus the function is log-convex. That is, for $l, r, s \in \mathcal{C}$ such that $\mathrm{r}<\mathrm{s}<\mathrm{l}$, the following holds

$$
\Lambda^{l-r}\left(g_{s}\right) \leqslant \Lambda^{l-s}\left(g_{r}\right) \Lambda^{s-r}\left(g_{l}\right) .
$$

Now we present different families of functions to investigate exponential convexity.

Remark 3.7. The following positive functionals are useful in defining the basic inequality of log-convex functions,

$$
\Lambda\left(f_{l}\right)=\frac{1}{l^{2}}(B(\exp (l A(h \cdot \omega)))-\exp (l A(h)))
$$




$$
\begin{aligned}
& \Lambda\left(g_{l}\right)=\frac{1}{l(l-1)}\left(B\left((A(h \cdot \omega))^{l}\right)-(A(h))^{l}\right), \\
& \Lambda\left(h_{l}\right)=\frac{1}{(\log l)^{2}}\left(B\left(l^{-A(h \cdot \omega)}\right)-l^{-A(h)}\right), \\
& \Lambda\left(k_{l}\right)=\frac{1}{l}(B(\exp (-A(h \cdot \omega) \sqrt{l}))-\exp (-A(h) \sqrt{l})) .
\end{aligned}
$$

Theorem 3.8. Let $\Lambda(\Psi)$ be the linear functional defined by (3.1) and consider the function $\phi_{i}:(0, \infty) \rightarrow \mathbb{R}$ for $i=1,4$ and $\phi_{i}:(1, \infty) \rightarrow \mathbb{R}$ for $i=2,3$ defined by $\phi_{1}(l)=\Lambda\left(f_{l}\right), \phi_{2}(l)=\Lambda\left(g_{l}\right), \phi_{3}(l)=\Lambda\left(h_{l}\right), \phi_{4}(l)=$ $\Lambda\left(k_{l}\right)$, where $f_{l}, g_{l}, h_{l}$, and $k_{l}$ are defined in Lemma 2.8. Then

(i) the functions $\phi_{i}$ are continuous on $(0, \infty)$ for $i=1,4$ and continuous on $(1, \infty)$ for $i=2,3$;

(ii) if $\mathrm{m} \in \mathbb{N}, l_{1}, \ldots, l_{\mathrm{m}} \in(0, \infty)$ for $i=1,4$ and $l_{1}, \ldots, l_{\mathrm{m}} \in(1, \infty)$ for $i=2,3$, then the matrices $\left[\phi_{i}\left(\frac{l_{j}+l_{k}}{2}\right)\right]_{j, k=1}^{m}$ are positive semidefinite;

(iii) the functions $\phi_{i}$ are exponentially convex on $(0, \infty)$ for $i=1,4$ and exponentially convex on $(1, \infty)$ for $i=2,3$;

(iv) let $l, r, s \in(0, \infty)$ for $i=1,4$ and $r, s, l \in(1, \infty)$ for $i=2,3$ with $l<r<s$, then

$$
\left(\phi_{i}(r)\right)^{s-l} \leqslant\left(\phi_{i}(l)\right)^{s-r}\left(\phi_{i}(s)\right)^{r-l},
$$

where $\phi_{i}(l)$ for $i=1,2,3,4$ are defined in Remark 3.7.

\subsection{Mean value theorems}

Now, we state the mean value theorems of Lagrange and Cauchy type.

Theorem 3.9. Let $\mathrm{L}$ satisfies properties $\mathrm{L}_{1}$ and $\mathrm{L}_{2}$ on a nonempty set $\mathrm{E}$, and let $\Psi: S=[\mathrm{a}, \mathrm{b}] \rightarrow \mathbb{R}, \Psi \in \mathrm{C}^{2}(\mathrm{~S})$, where $S \subseteq \mathbb{R}$ is a compact real interval. If $\mathrm{A}$ and $\mathrm{B}$ are positive linear functionals with $\mathrm{A}(1)=\mathrm{B}(1)=1$, and $\omega$ is a weight function (defined in (1.3)) then for all $h, h \cdot \omega \in L$ such that $\Psi(A(h \cdot \omega)),(A(h \cdot \omega))^{2} \in L$ there exists some $\varpi \in \mathrm{S}$ such that the following holds

$$
B(\Psi(A(h \cdot \omega)))-\Psi(A(h))=\frac{\Psi^{\prime \prime}(\varpi)}{2}\left[B\left((A(h \cdot \omega))^{2}\right)-(A(h))^{2}\right] .
$$

Theorem 3.10. Let $\mathrm{L}$ satisfies properties $\mathrm{L}_{1}$ and $\mathrm{L}_{2}$ on a nonempty set $\mathrm{E}$, and let $\Phi, \Psi: S=[\mathrm{a}, \mathrm{b}] \rightarrow \mathbb{R}, \Phi, \Psi \in$ $\mathrm{C}^{2}(S)$, where $S \subseteq \mathbb{R}$ is a compact real interval. If $\mathrm{A}$ and $\mathrm{B}$ are positive linear functionals with $\mathrm{A}(1)=1$ and $B(1)=1$, and $\omega$ is a weight function (defined in (1.3)), then for all $h, h \cdot \omega \in L$ such that $\Phi(A(h \cdot \omega)), \Psi(A(h$. $\omega),(A(h \cdot \omega))^{2} \in L$ and $B\left((A(h \cdot \omega))^{2}\right)-(A(h))^{2} \neq 0$, there exists some $\Phi \in S$ such that the following holds

$$
\Psi^{\prime \prime}(\varpi)[B(\Phi(A(h \cdot \omega)))-\Phi(A(h))]=\Phi^{\prime \prime}(\varpi)[B(\Psi(A(h \cdot \omega)))-\Psi(A(h))] .
$$

Remark 3.11. We can construct similar results for the positive functional $\neg(\Psi)$ defined in (3.2).

\section{Acknowledgment}

This research is supported by National University of Sciences and Technology (NUST), Islamabad and Higher Education Commission of Pakistan.

\section{References}

[1] M. Anwar, N. Latif, J. Pečarić, Positive semidefinite matrices, exponential convexity for majorization, and related Cauchy means, J. Inequal. Appl., 2010 (2010), 19 pages. 1

[2] S. I. Butt, R. Jakšić, L. Kvesić, J. Pečarić, n-Exponential convexity of weighted Hermite-Hadamard's inequality, J. Math. Inequal., 8 (2014), 299-311. 1

[3] S. S. Dragomir, J. Pečarić, L. E. Persson, Properties of some functionals related to Jensen's inequality, Acta Math. Hungar., 70 (1996), 129-143. 1 
[4] S. Iqbal, K. Krulić Himmelreich, J. Pečarić, D. Pokaz, n-Exponential convexity of Hardy-type and Boas-type functionals, J. Math. Inequal., 7 (2103), 739-750. 1

[5] J. Jakšetić, R. Naeem, J. Pečarić, Exponential convexity for Jensen's inequality for norms, J. Inequal. Appl., 2016 (2016), 8 pages. $1,2.3$

[6] J. Jakšetić, J. Pečarić, Exponential Convexity method, J. Convex Anal., 20 (2013), 181-197. 1, 2, 2

[7] B. Jessen, Bemaerkinger om konvekse Funktioner og Uligheder imellem Middelvaerdier I, Mat. Tidsskrift B, 1931 (1931), 17-28. 1

[8] S. Khalid, J. Pečarić, On the refinements of the integral Jensen-Steffensen inequality, J. Inequal. Appl., 2013 (2013), 18 pages. 1

[9] K. A. Khan, A. Nosheen, J. Pečarić, n-Exponential convexity of some dynamic Hardy-type functionals, J. Math. Inequal., 8 (2014), 331-347.

[10] A. R. Khan, J. Pečarić, M. Rodić Lipanović, n-Exponential convexity for Jensen-type inequalities, J. Math. Inequal., 7 (2013), 313-335. 1

[11] D. S. Mitrinović, J. E. Pečarić, A. M. Fink, Classical and New Inequalities in Analysis, Kluwer Academic Publishers Group, Dordrecht, (1993). 1

[12] R. Naeem, m-Exponential convexity of refinements of Hermite-Hadamards inequality, Proc. Pak. Acad. Sci. A, 54 (2017), 197-205. 1

[13] R. Naeem, M. Anwar, Jessen type functionals and exponential convexity, J. Math. Computer Sci., 17 (2017), 429-436. 1

[14] J. Pečarić, J. Perić, Improvement of the Giaccardi and the Petrović inequality and related Stolarsky type means, An. Univ. Craiova Ser. Mat. Inform., 39 (2012), 65-75. 1, 2, 2

[15] J. E. Pečarić, F. Proschan, Y. L. Tong, Convex functions, partial orderings, and statistical applications, Academic Press, Boston, (1992). 1, 1, 2.11

[16] J. Rooin, A refinement of Jensen's inequality, J. Inequal. Pure Appl. Math., 6 (2005), 4 pages. 1.1

[17] J. Rooin, Some refinements of discrete Jensen's inequality and some of its applications, Nonlinear Funct. Anal. Appl., 12 (2007), 107-118. 2.15

[18] W. Rudin, Real and complex analysis, McGraw-Hill Book Co., New York, (1966). 1 